Bull. Korean Math. Soc. 51 (2014), No. 3, pp. 641-651

http://dx.doi.org/10.4134/BKMS.2014.51.3.641

\title{
CONVEX SOLUTIONS OF THE POLYNOMIAL-LIKE ITERATIVE EQUATION ON OPEN SET
}

\author{
XiaObing Gong
}

\begin{abstract}
Because of difficulty of using Schauder's fixed point theorem to the polynomial-like iterative equation, a lots of work are contributed to the existence of solutions for the polynomial-like iterative equation on compact set. In this paper, by applying the Schauder-Tychonoff fixed point theorem we discuss monotone solutions and convex solutions of the polynomial-like iterative equation on an open set (possibly unbounded) in $\mathbb{R}^{N}$. More concretely, by considering a partial order in $\mathbb{R}^{N}$ defined by an order cone, we prove the existence of increasing and decreasing solutions of the polynomial-like iterative equation on an open set and further obtain the conditions under which the solutions are convex in the order.
\end{abstract}

\section{Introduction}

As indicated in the books $[7,19]$ and the surveys $[3,24]$, the polynomial-like iterative equation

$$
\lambda_{1} f(x)+\lambda_{2} f^{2}(x)+\cdots+\lambda_{n} f^{n}(x)=F(x), \quad x \in S,
$$

where $S$ is a subset of a linear space over $\mathbb{R}, F: S \rightarrow S$ is a given function, $\lambda_{i} \mathrm{~s}(i=1, \ldots, n)$ are real constants, $f: S \rightarrow S$ is the unknown function and $f^{i}$ is the $i$ th iterate of $f$, i.e., $f^{i}(x)=f\left(f^{i-1}(x)\right)$ and $f^{0}(x)=x$ for all $x \in S$, is one of important forms of functional equation since it is the basic form of iterative functional equation and the problem of iterative roots and the problem of invariant curves can be reduced to the kind of equations. For $S \subset \mathbb{R}$, while some works (e.g. $[4,6,12,13,14,17,22]$ ) are contributed to the case of linear $F$, there are given many results to the case of nonlinear $F$, for example, [11, 29] for $n=2$, [25] for general $n$, [10, 26] for smoothness, [16] for analyticity. Some efforts were also devoted to Eq.(1.1) in high-dimensional spaces such as in [27], radially monotonic solutions were discussed in highdimensional Euclidean spaces by properties of orthogonal group. Later, also in

Received May 25, 2012; Revised May 10, 2013.

2010 Mathematics Subject Classification. 39B12, 58F08.

Key words and phrases. iterative equation, open set, order, increasing operator and decreasing operator.

Supported by Key Project of Sichuan Provincial Department of Education (12ZA086). 
high-dimensional Euclidean spaces, the existence of Lipschitzian solutions were investigated for the case $n=\infty$ on a compact convex subset of $\mathbb{R}^{N}, N>1$ in [9]. In 2004, [9] were partially generalized to an arbitrary closed (not necessarily convex) subset of a Banach space in [18].

Convexity is one of important properties of functions and is often used in optimization, mathematical programming and game theory. The study of convexity for iterative equations can be traced to 1968, when Kuczma and Smajdor [8] investigated the convexity of iterative roots. Some recent results can be found from $[20,21,28]$ in 1-dimensional space and in high-dimensional spaces one can refer to [5]. In [28], convex solutions and concave ones of Eq.(1.1) were discussed under the normalization condition: $\sum_{j=1}^{n} \lambda_{j}=1$ on a compact interval. More concretely, the existence and uniqueness of convex (resp. concave) solutions with uniform non-positiveness of $\lambda_{2}, \ldots, \lambda_{n}$ and increasing convex (resp. concave) with uniform non-negativeness of $\lambda_{2}, \ldots, \lambda_{n}$ are proved. And as a continuation of [28], increasing convex (or concave) solutions and decreasing convex (or concave) solutions of Eq.(1.1) are completely investigated with no normalization condition and no requirement of uniform sign of coefficients on a compact interval in [21]. In [20], nondecreasing convex solutions for Eq.(1.1) on open intervals (possibly unbounded) were discussed. For high-dimensional spaces, by considering a partial order, which is defined by an order cone, the existence of increasing convex (concave) solutions of Eq.(1.1) on a compact subset of Banach spaces were proved in [5].

In this paper, motivated by [20], we consider Eq.(1.1) on an open set (possibly unbounded) in $\mathbb{R}^{N}$. Through defining a separating family of seminorms on a continuous function space which becomes a locally convex space and considering compact convergence topology on this locally convex function space and by applying the Schauder-Tychonoff fixed point theorem (see the Appendix), we discuss monotone solutions and convex solutions of polynomial-like iterative equation on an open set in $\mathbb{R}^{N}$. More concretely, as in [5], we consider a partial order in $\mathbb{R}^{N}$, which is defined by an order cone, and give existence of increasing solutions and decreasing solutions in this ordered space. Then we further give conditions under which those solutions are convex or concave.

\section{Preliminaries}

As indicated in the Introduction, in order to discuss monotonicity and convexity of solutions for Eq.(1.1) on an open set in $\mathbb{R}^{N}$, we need to introduce an order in $\mathbb{R}^{N}$. For convenience, we use the conventions of [5].

As in [23], a nonempty subset $K$ of a real vector space $X$ is called a cone if $x \in K$ implies that $a x \in K$ for all $a>0$. A nonempty and nontrivial subset $K \subset X$ is an order cone if $K$ is a convex cone and satisfies $K \cap(-K)=\{\theta\}$, where $\theta$ denotes the zero element of $X$. Having chosen such an order cone $K$ in $X$, we can define a partial order $x \leq_{K} y$ in $X$, simply called the $K$-order, if

$$
y-x \in K \text {. }
$$


After introducing a $K$-order, one can define ordered vector spaces, ordered real Banach spaces and normal order cones as in [2]. A real vector space $X$ equipped with a $K$-order is called an ordered vector space, denoted by $(X, K)$. A real Banach space $(X,\|\cdot\|)$ associated with a $K$-order is called an ordered real Banach space, denoted by $(X, K,\|\cdot\|)$, if $K$ is closed. An order cone $K$ in an ordered real Banach space $(X, K,\|\cdot\|)$ is said to be normal if there exists a constant $N>0$ such that $\|x\| \leq N\|y\|$ if $\theta \leq_{K} x \leq_{K} y$ in $X$. The smallest constant $N$, denoted by $N(K)$, is called the normal constant of $K$.

In an ordered real vector space $(X, K)$ one can define increasing (decreasing) operators and convex (concave) operators as in [2]. An operator $f: D \subset X \rightarrow$ $X$ is said to be increasing (resp. decreasing) in the sense of the $K$-order if $x \leq_{K} y$ implies $f(x) \leq_{K} f(y)$ (resp. $f(x) \geq_{K} f(y)$ ). An operator $f: D \rightarrow X$, where $D \subset X$ is a convex subset, is said to be convex (resp. concave) in the sense of the $K$-order if $f(\lambda x+(1-\lambda) y) \leq_{K} \lambda f(x)+(1-\lambda) f(y)$ (resp. $\left.f(\lambda x+(1-\lambda) y) \geq_{K} \lambda f(x)+(1-\lambda) f(y)\right)$ for all $\lambda \in[0,1]$ and for every pair of distinct comparable points $x, y \in D$ (i.e., either $x \leq_{K} y$ or $x \geq_{K} y$ ).

Let $\Omega$ be an open convex subset of $\left(\mathbb{R}^{N}, K,\|\cdot\|\right)$ with nonempty interior, where $\|\cdot\|$ is Euclidean norm and $C\left(\Omega, \mathbb{R}^{N}\right)$ consists of all continuous functions $f: \Omega \rightarrow \mathbb{R}^{N}$. Then there exists a sequence $\Omega_{1}, \Omega_{2}, \ldots$ of compact subsets of $\Omega$ such that

$$
\Omega=\bigcup_{n=1}^{\infty} \Omega_{n} \text { and } \Omega_{n} \subset \operatorname{int} \Omega_{n+1} \text { for every } n \in \mathbb{N} .
$$

For every positive integer $n$, let $p_{n}: C\left(\Omega, \mathbb{R}^{N}\right) \rightarrow \mathbb{R}$ be the function defined by

$$
p_{n}(f):=\sup _{x \in \Omega_{n}}\|f(x)\| \text {. }
$$

Then $\mathcal{P}:=\left(p_{n}\right)_{n \in \mathbb{N}}$ is a separating family of seminorms on the vector space $C\left(\Omega, \mathbb{R}^{N}\right)$. Since $p_{1} \leq p_{2} \leq \cdots$, in accordance with Theorem 1.37 in [15], the sets

$$
V_{n}=\left\{f \in C(\Omega, X): p_{n}(f)<\frac{1}{n}\right\}(n=1,2,3, \ldots)
$$

form a convex local base for $C\left(\Omega, \mathbb{R}^{N}\right)$, which turns $C\left(\Omega, \mathbb{R}^{N}\right)$ into a locally convex topological space. According to remark (c) of Section 1.38 in [15], the topology of $C\left(\Omega, \mathbb{R}^{N}\right)$ is compatible with the metric

$$
d(f, g)=\max _{n \in \mathbb{N}} \frac{2^{-n} p_{n}(f-g)}{1+p_{n}(f-g)} .
$$

Moreover, the metrizable locally convex space $\left(C\left(\Omega, \mathbb{R}^{N}\right), d\right)$ is complete. We now prove that this topology of $C\left(\Omega, \mathbb{R}^{N}\right)$ is compact convergence topology. We need the following lemma.

Lemma 2.1. Let $S$ be a subset of a topology space. Let $S=\bigcup_{n=1}^{\infty} S_{n}$ and $S_{n} \subset \operatorname{int} S_{n+1}$ for every $n \in \mathbb{N}$. Then for every compact subset $A$ of $S$, there exists a $N \in \mathbb{N}$ such that $A \subset S_{N}$. 
Proof. If $A \nsubseteq S_{n}$, then for $n \in \mathbb{N}$ there exists $x_{n} \in A$ such that $x_{n} \notin S_{n}$. Hence we have a sequence $\left\{x_{n}\right\}$ such that $\left\{x_{n}\right\} \subseteq A$ and $x_{n} \notin S_{n}$ for every $n \in \mathbb{N}$. Compactness of $A$ implies that $\left\{x_{n}\right\}$ has a convergence subsequence $\left\{x_{n_{k}}\right\}$. Let $\lim _{k \rightarrow \infty} x_{n_{k}}=x_{0}$, then $x_{0} \in A$. Hence $x_{0} \in S$. Since $S=\bigcup_{n=1}^{\infty} S_{n}$, there exists a $N \in \mathbb{N}$ such that $x_{0} \in S_{N}$. Consequently, $x_{0} \in \operatorname{int} S_{N+1}$. So there exists a neighbourhood $U\left(x_{0}\right)$ of $x_{0}$ such that there exists $x_{n_{J}} \in U\left(x_{0}\right)$ $\left(n_{J}>N+1\right)$ and $U\left(x_{0}\right) \subseteq S_{N+1}$. So $x_{n_{J}} \in S_{N+1}$, which contradicts with $x_{n_{J}} \notin S_{n_{J}}$. We complete the proof.

Suppose $\left\{f_{j}\right\} \subseteq C\left(\Omega, \mathbb{R}^{N}\right)$ and $f_{j} \rightarrow f \in C\left(\Omega, \mathbb{R}^{N}\right)(j \rightarrow \infty)$ with respect to metric $d$, then for every integer $n, p_{n}\left(f_{j}-f\right) \rightarrow 0(j \rightarrow \infty)$. By Lemma 2.1, $\left\{f_{j}\right\}$ converges uniformly to $f$ on every compact subset of $\Omega$. Conversely, if $\left\{f_{j}\right\} \subseteq C\left(\Omega, \mathbb{R}^{N}\right)$ converges uniformly to $f \in C\left(\Omega, \mathbb{R}^{N}\right)$ on every compact subset of $\Omega$, then $\left\{f_{j}\right\}$ converges uniformly to $f$ on $S_{n}(n \in \mathbb{N})$. This implies that $f_{j} \rightarrow f(j \rightarrow \infty)$ with respect to metric $d$. Consequently, the topology of $C\left(\Omega, \mathbb{R}^{N}\right)$ is compact convergence topology.

Let $\Omega_{0} \subset \Omega$ be a given compact convex subset of $\mathbb{R}^{N}$. For $0 \leq m \leq M<$ $+\infty$, define

$$
\begin{aligned}
C^{+}(\Omega, m, M):= & \left\{f \in C\left(\Omega, \mathbb{R}^{N}\right): f(\Omega) \subset \Omega_{0} \subset \Omega,\right. \\
& m(y-x) \leq_{K} f(y)-f(x) \leq_{K} M(y-x) \text { if } x \leq_{K} y, \text { and } \\
& \|f(y)-f(x)\| \leq M\|y-x\| \text { if } x \text { and } y \text { are not comparable }\}, \\
C^{-}(\Omega, m, M):= & \left\{f \in C\left(\Omega, \mathbb{R}^{N}\right): f(\Omega) \subset \Omega_{0} \subset \Omega,\right. \\
& m(y-x) \leq_{K} f(x)-f(y) \leq_{K} M(y-x) \text { if } x \leq_{K} y, \text { and } \\
& \|f(y)-f(x)\| \leq M\|y-x\| \text { if } x \text { and } y \text { are not comparable }\}, \\
C_{c v}^{+}(\Omega, m, M):= & \left\{f \in C^{+}(\Omega, m, M): f \text { is convex on } \Omega \text { in } K \text {-order }\right\}, \\
C_{c c}^{+}(\Omega, m, M):= & \left\{f \in C^{+}(\Omega, m, M): f \text { is concave on } \Omega \text { in } K \text {-order }\right\}, \\
C_{c v}^{-}(\Omega, m, M):= & \left\{f \in C^{-}(\Omega, m, M): f \text { is convex on } \Omega \text { in } K \text {-order }\right\}, \\
C_{c c}^{-}(\Omega, m, M):= & \left\{f \in C^{-}(\Omega, m, M): f \text { is concave on } \Omega \text { in } K \text {-order }\right\} .
\end{aligned}
$$

Lemma 2.2. Let $\left(\mathbb{R}^{N}, K,\|\cdot\|\right)$ be an ordered real Banach space such that $K$ is normal. Then the above defined $C^{+}(\Omega, m, M), C^{-}(\Omega, m, M), C_{c v}^{+}(\Omega, m, M)$ and $C_{c c}^{+}(\Omega, m, M)$ are compact convex subsets of $C\left(\Omega, \mathbb{R}^{N}\right)$ with respect to compact convergence topology for any $0 \leq m \leq M<+\infty$.

Proof. We only prove that $C^{+}(\Omega, m, M)$ and $C_{c v}^{+}(\Omega, m, M)$ are compact convex subsets of $C\left(\Omega, \mathbb{R}^{N}\right)$ with respect to compact convergence topology. It will be similar for $C^{-}(\Omega, m, M)$ and $C_{c c}^{+}(\Omega, m, M)$. We first consider $C^{+}(\Omega, m, M)$. Let $\Xi$ be any compact subset of $\Omega$ and $\left\{f_{i}\right\} \subset C^{+}(\Omega, m, M)$. We prove that $\left\{f_{i}\right\}$ has a subsequence which is uniformly convergent on $\Xi$. By Lemma 2.1, it is sufficient to claim it on every $\Omega_{n}(n=1,2, \ldots)$. We use diagonal argument to prove it. Similar to Lemma 2.2 in [5] one can prove that $\left\{f_{i}\right\}$ has a convergent subsequence with respect to sup norm on $\Omega_{1}$ by Ascoli's Theorem, 
which is denoted by $\left.\left\{f_{1, k}\right\}\right|_{k=1} ^{\infty}$. Similarly, $\left.\left\{f_{1, k}\right\}\right|_{k=1} ^{\infty}$ has a convergent subsequence $\left.\left\{f_{2, k}\right\}\right|_{k=1} ^{\infty}$ with respect to sup norm on $\Omega_{2}$. Proceeding in this fashion we obtain a countable collection of subsequences of the original sequence:

$$
\begin{array}{cccccc}
f_{1,1} & f_{1,2} & f_{1,3} & \ldots & f_{1, k} & \ldots, \\
f_{2,1} & f_{2,2} & f_{2,3} & \ldots & f_{2, k} & \ldots, \\
\cdot & \cdot & \cdot & \ldots & \cdot & \ldots, \\
f_{n, 1} & f_{n, 2} & f_{n, 3} & \ldots & f_{n, k} & \ldots, \\
\cdot & \cdot & \cdot & \ldots & \cdot & \ldots,
\end{array}
$$

where the sequence in the $n$-th row is uniform convergence on $\Omega_{n}$, and each row is a subsequence of the one above it. Thus the diagonal sequence $\left\{f_{n, n}\right\}_{n=1}^{\infty}$ is a subsequence of the original sequence $\left\{f_{i}\right\}$ and $\left.\left\{f_{n, n}\right\}\right|_{n=1} ^{\infty}$ is uniform convergence on $\Omega_{j}(j=1,2, \ldots)$. In fact, for any $\Omega_{j},\left.\left\{f_{j, k}\right\}\right|_{k=1} ^{\infty}$ is uniform convergence on $\Omega_{j}$ and $\left.\left\{f_{n, n}\right\}\right|_{n=j} ^{\infty}$ is the subsequence of $\left.\left\{f_{j, k}\right\}\right|_{k=1} ^{\infty}$. Hence $\left.\left\{f_{n, n}\right\}\right|_{n=j} ^{\infty}$ is uniform convergence on $\Omega_{j}$, which implies that $\left.\left\{f_{n, n}\right\}\right|_{n=1} ^{\infty}$ uniformly converge on $\Omega_{j}(j=1,2, \ldots)$. Consequently, we have proved that $C^{+}(\Omega, m, M)$ is relatively compact with respect to compact convergence topology.

Furthermore, we prove that $C^{+}(\Omega, m, M)$ is a closed subset of $C\left(\Omega, \mathbb{R}^{N}\right)$ with respect to compact convergence topology. Let $\left\{f_{i}\right\} \subset C^{+}(\Omega, m, M)$ be such a sequence that $\lim _{i \rightarrow \infty} f_{i}=f$ in $C\left(\Omega, \mathbb{R}^{N}\right)$, i.e., $\left\{f_{i}\right\}$ converges uniformly on $\Omega_{n}$ to $f$. Hence

$$
\lim _{i \rightarrow \infty}\left\|f_{i}(x)-f(x)\right\|=0, \forall x \in \Omega_{n}, n=1,2, \ldots
$$

For any $x, y \in \Omega$, there exists $\Omega_{j}$ such that $x, y \in \Omega_{j}$. Similar to Lemma 2.2 in [5] and by (2.1), we have

$$
m(y-x) \leq_{K} f(y)-f(x) \leq_{K} M(y-x)
$$

if $x \leq_{K} y$ and

$$
\|f(y)-f(x)\| \leq M\|y-x\|
$$

if $x$ and $y$ are not comparable. At last, we can prove that $f(\Omega) \subset \Omega_{0} \subset \Omega$ because $f_{i}(\Omega) \subset \Omega_{0}$ and $\Omega_{0}$ is a compact set. Obviously, $f \in C\left(\Omega, \mathbb{R}^{N}\right)$. Thus, we have proved that $C^{+}(\Omega, m, M)$ is a closed set and therefore a compact subset of $C\left(\Omega, \mathbb{R}^{N}\right)$.

The proof of convexity of $C^{+}(\Omega, m, M)$ is similar to Lemma 2.2 in [5]. So we have proved that $C^{+}(\Omega, m, M)$ is a compact convex subset of $C\left(\Omega, \mathbb{R}^{N}\right)$.

The proof for $C_{c v}^{+}(\Omega, m, M)$ is similar to Lemma 2.2 in [5]. The proof is completed.

Lemma 2.3. Let $Y$ be the set consisting of all functions $f \in C\left(\Omega, \mathbb{R}^{N}\right)$ such that $f(\Omega) \subseteq \Omega$ and $f$ is Lipschitz on $\Omega$. Furthermore, let $F \in C\left(\Omega, \mathbb{R}^{N}\right)$, let $\alpha_{1}, \alpha_{2}, \ldots, \alpha_{n} \in \mathbb{R}$, and let $L: Y \rightarrow C\left(\Omega, \mathbb{R}^{N}\right)$ be the operator defined by

$$
(L f)(x):=\alpha_{1} F(x)+\alpha_{2} f^{2}(x)+\cdots+\alpha_{n} f^{n}(x)
$$

for every $f \in Y$ and each $x \in \Omega$. Then $L$ is continuous on $Y$. 
Proof. Fix any $f \in Y$ and let $V$ be any neighbourhood of $L f$ in $C\left(\Omega, \mathbb{R}^{N}\right)$. Choose $l \in \mathbb{N}$ such that $L f+V_{l} \subseteq V$. Since $f$ is continuous and $\Omega_{l}$ is a compact subset of $\Omega$, it follows that $f^{j}\left(\Omega_{l}\right)$ is a compact subset of $\Omega$ for every $j \in\{0,1, \ldots, n\}$. Set $E=\bigcup_{j=0}^{n} f^{j}\left(\Omega_{l}\right)$. By Lemma 2.1, there exists $\Omega_{m}$ such that $E \subseteq \Omega_{m}$. Let $\lambda \geq 0$ be the Lipschitz constant of $f$ on $\Omega$. Let $r=\max \{m, k\}$, where $k$ is a integer such that $k>l \sum_{j=2}^{n}\left|\alpha_{j}\right| \sum_{i=0}^{j-1} \lambda^{i}$. Let $U:=\left(f+V_{r}\right) \bigcap Y$. Clearly, $U$ is a neighbourhood of $f$ in $Y$. We will prove $L(U) \subseteq L f+V_{l}$. For any $g \in U, L g \in C\left(\Omega, \mathbb{R}^{N}\right)$ and $p_{r}(f-g)<\frac{1}{r}$. Note that $\Omega_{l} \subseteq \Omega_{r}$. Hence

$$
\begin{aligned}
p_{l}((L f)-(L g)) & \leq p_{r}((L f)-(L g)) \\
& \leq \frac{1}{r} \sum_{j=2}^{n}\left|\alpha_{j}\right| \sum_{i=0}^{j-1} \lambda^{i} \\
& \leq \frac{1}{k} \sum_{j=2}^{n}\left|\alpha_{j}\right| \sum_{i=0}^{j-1} \lambda^{i} \\
& \leq \frac{1}{l}, \forall x \in \Omega_{l} .
\end{aligned}
$$

Consequently, $L g \in L f+V_{l}$, which implies $L(U) \subseteq V$. We complete the proof of continuity of $L$ at $f$.

\section{Increasing and decreasing solutions}

Before discussing convexity, we prove the existence of increasing and decreasing solutions of Eq.(1.1) in the ordered real Banach space $\left(\mathbb{R}^{N}, K,\|\cdot\|\right)$ such that $K$ is normal and $N(K) \leq 1$. First, we investigate increasing solutions. Consider Eq.(1.1) with the following hypotheses:

(H1) $\lambda_{1}>0, \lambda_{i} \leq 0, i=2,3, \ldots, n$, and

(H2) the normalization condition $\sum_{i=1}^{n} \lambda_{i}=1$.

Theorem 3.1. Suppose that (H1) and (H2) hold and $F \in C^{+}\left(\Omega, 0, M_{1}\right)$, where $M_{1} \in(0,+\infty)$ is a constant. If

$$
M_{1} \leq \lambda_{1} M+\lambda_{2} M^{2}+\cdots+\lambda_{n} M^{n}
$$

for a constant $M \in[0,+\infty)$, then Eq.(1.1) has a solution $f \in C^{+}(\Omega, 0, M)$.

Proof. Under the hypothesis (H1) and (H2), we can rewrite Eq.(1.1) as

$$
f(x)=\frac{1}{\lambda_{1}} F(x)-\frac{\lambda_{2}}{\lambda_{1}} f^{2}(x)-\cdots-\frac{\lambda_{n}}{\lambda_{1}} f^{n}(x), x \in \Omega,
$$

where

$$
\frac{1}{\lambda_{1}}-\sum_{i=2}^{n} \frac{\lambda_{i}}{\lambda_{1}}=1
$$


Define a mapping $L: C^{+}(\Omega, 0, M) \rightarrow C\left(\Omega, \mathbb{R}^{N}\right)$ by

$$
L f(x)=\frac{1}{\lambda_{1}} F(x)-\frac{\lambda_{2}}{\lambda_{1}} f^{2}(x)-\cdots-\frac{\lambda_{n}}{\lambda_{1}} f^{n}(x) .
$$

We first prove that $L$ satisfies $L\left(C^{+}(\Omega, 0, M)\right) \subset A \subset C^{+}(\Omega, 0, M)$ with $A$ compact. Similar to Lemma 2.2 in [5], we have

$$
\|f(x)-f(y)\| \leq M_{0}\|x-y\|, \quad \forall x, y \in \Omega, \quad \forall f \in C^{+}(\Omega, 0, M),
$$

which implies that $f$ is Lipschitz on $\Omega$, where $M_{0}=\max \{M, M N(K)\}$. By definition of $C^{+}(\Omega, 0, M), f(\Omega) \subset \Omega$. Hence, by Lemma $2.3 L$ is continuous on $C^{+}(\Omega, 0, M)$. By inequality (3.1), similar to Theorem 3.1 in [5], $L\left(C^{+}(\Omega, 0, M)\right) \subset C^{+}(\Omega, 0, M)$. As Lemma 2.2 guarantees that $C^{+}(\Omega, 0, M)$ is a convex subset of the Hausdorff locally convex linear topological space $C\left(\Omega, \mathbb{R}^{N}\right)$, by Schauder-Tychonoff fixed point theorem we see that $L$ has a fixed point $f \in C^{+}(\Omega, 0, M)$. Thus $f$ is an increasing solution of the equation. The proof is completed.

The following is devoted to decreasing solutions. The proof is similar to Theorem 3.1 by using the same arguments as in Theorem 3.2 in [5], we only show the result but omit it.

Theorem 3.2. Suppose that (H1) and (H2) hold and all coefficients of even order iterates in Eq.(1.1) are equal to 0 . Let $F \in C^{-}\left(\Omega, 0, M_{1}\right)$, where $M_{1} \in$ $(0,+\infty)$ is a constant. If the condition (3.1) holds for a constant $M \in(0,+\infty)$, then Eq.(1.1) has a solution $f \in C^{-}(\Omega, 0, M)$.

\section{Convexity of solutions}

On the basis of last section we can discuss on convexity of continuous solutions for Eq.(1.1) in the ordered real Banach space $\left(\mathbb{R}^{N}, K,\|\cdot\|\right)$ with a normal cone $K$ and $N(K) \leq 1$.

Theorem 4.1. Suppose that $(\mathrm{H} 1)$ and $(\mathrm{H} 2)$ hold and $F \in C_{c v}^{+}\left(\Omega, 0, M_{1}\right)$, where $M_{1} \in(0,+\infty)$ is a constant. If

$$
M_{1} \leq \lambda_{1} M+\lambda_{2} M^{2}+\cdots+\lambda_{n} M^{n}
$$

for a constant $M \in(0,+\infty)$, then Eq.(1.1) has a continuous solution $f \in$ $C_{c v}^{+}(\Omega, 0, M)$.

Proof. Define a mapping $L: C_{c v}^{+}(\Omega, 0, M) \rightarrow C\left(\Omega, \mathbb{R}^{N}\right)$ as in Theorem 3.1. The continuity of $L$ was proved in the proof of Theorem 3.1. Use the same argument as in Theorem 4.1 in [5] we can prove $L\left(C_{c v}^{+}(\Omega, 0, M)\right) \subset C_{c v}^{+}(\Omega, 0, M)$. Lemma 2.2 guarantees that $C_{c v}^{+}(\Omega, 0, M)$ is a compact convex subset of the Hausdorff locally convex linear topological space $C\left(\Omega, \mathbb{R}^{N}\right)$. Therefore, this proof can be completed by using Schauder-Tychonoff fixed point theorem.

Similarly, we can prove the following result for concavity of solutions. 
Theorem 4.2. Suppose that (H1) and (H2) hold and $F \in C_{c c}^{+}\left(\Omega, 0, M_{1}\right)$, where $M_{1} \in(0,+\infty)$ is a constant. If

$$
M_{1} \leq \lambda_{1} M+\lambda_{2} M^{2}+\cdots+\lambda_{n} M^{n}
$$

for a constant $M \in(0,+\infty)$, then Eq.(1.1) has a continuous solution $f \in$ $C_{c c}^{+}(\Omega, 0, M)$.

Example 4.1. Consider the equation

(4.3) $\frac{10}{9} f\left(x_{1}, x_{2}\right)-\frac{1}{9} f^{2}\left(x_{1}, x_{2}\right)=\left(\frac{-1+2 x_{1}}{1-x_{1}}, \frac{-47+16 x_{2}}{6-2 x_{2}}\right), \forall\left(x_{1}, x_{2}\right) \in \Omega$, where $\Omega:=\left\{\left(x_{1}, x_{2}\right) \in \mathbb{R}^{2}: x_{1}<0, x_{2}<0\right\}$. Clearly, Eq.(4.3) is of the form (1.1), where

$$
F\left(x_{1}, x_{2}\right):=\left(\frac{-1+2 x_{1}}{1-x_{1}}, \frac{-47+16 x_{2}}{6-2 x_{2}}\right),
$$

$\lambda_{1}=10 / 9$ and $\lambda_{2}=-1 / 9 . \quad \Omega$ is a open convex subset (unbounded) of the ordered real Banach space $\left(\mathbb{R}^{2}, K,\|\cdot\|\right)$, where $\|x\|=\left(x_{1}^{2}+x_{2}^{2}\right)^{1 / 2}$ and $K:=$ $\left\{x=\left(x_{1}, x_{2}\right): x_{1} \geq 0, x_{2} \geq 0\right\}$ is a normal order cone and $N(K) \leq 1$. One can check that (H1) and (H2) are satisfied. We further claim that $F \in C_{c v}^{+}(\Omega, 0,1)$. Let

$$
g(t)=\frac{-1+2 t}{1-t}, h(t)=\frac{-47+16 t}{6-2 t}, \quad t \in(-\infty, 0) .
$$

By simple calculation we have

$$
g^{\prime}(t)=\frac{1}{(1-t)^{2}}, t \in(-\infty, 0)
$$

and

$$
\max _{t \in(-\infty, 0)}\left|g^{\prime}(t)\right| \leq 1
$$

Hence $\left|g\left(y_{1}\right)-g\left(x_{1}\right)\right| \leq\left|y_{1}-x_{1}\right|$ for all $x_{1}, y_{1} \in(-\infty, 0)$. Similarly, $\mid h\left(y_{2}\right)-$ $h\left(x_{2}\right)\left|\leq \frac{1}{18}\right| y_{2}-x_{2} \mid$ for all $x_{2}, y_{2} \in(-\infty, 0)$. Consequently,

$$
\begin{aligned}
\|F(y)-F(x)\| & =\sqrt{\left(g\left(y_{1}\right)-g\left(x_{1}\right)\right)^{2}+\left(h\left(y_{2}\right)-h\left(x_{2}\right)\right)^{2}} \\
& \leq \sqrt{\left(y_{1}-x_{1}\right)^{2}+\frac{1}{324}\left(y_{2}-x_{2}\right)^{2}} \\
& \leq\|y-x\|, \forall x, y \in \Omega,
\end{aligned}
$$

which implies that $F \in C\left(\Omega, \mathbb{R}^{N}\right)$. (4.4) implies that $g$ is strictly increasing on $(-\infty, 0)$ and

$$
\lim _{t \rightarrow-\infty} g(t)=-2, \lim _{t \rightarrow 0^{-}} g(t)=-1 .
$$

Hence $g((-\infty, 0)) \subset(-2,-1)$. Similarly, $h((-\infty, 0)) \subset(-8,-47 / 6)$. So $F(\Omega) \subset \Omega_{0}:=[-2,-1] \times[-8,-7] \subset \Omega$, where $\Omega_{0}$ is obviously a compact convex subset of $\mathbb{R}^{2}$. Note that $x \leq_{K} y$ if and only if $y_{1}-x_{1} \geq 0$ and 
$y_{2}-x_{2} \geq 0$. Thus, when $x, y \in \Omega$ are not comparable (i.e., $y-x \notin K$ and $x-y \notin K)$, we have (4.5); when $x, y \in \Omega$ are comparable, assume $x \leq_{K} y$, then $x_{1} \leq y_{1}, x_{2} \leq y_{2}$. Hence $0 \leq g\left(y_{1}\right)-g\left(x_{1}\right) \leq y_{1}-x_{1}$ and $0 \leq h\left(y_{2}\right)-h\left(x_{2}\right) \leq \frac{1}{18}\left(y_{2}-x_{2}\right)$ because $g(t), h(t)$ are strictly increasing on $(-\infty, 0)$ and $\max _{t \in(-\infty, 0)}\left|g^{\prime}(t)\right| \leq 1, \max _{t \in(-\infty, 0)}|h \prime(t)| \leq 1 / 18$. We calculate

$$
F(y)-F(x)=\left(g\left(y_{1}\right)-g\left(x_{1}\right), h\left(y_{2}\right)-h\left(x_{2}\right)\right), \forall x, y \in \Omega,
$$

implying that

$$
\theta \leq_{K} F(y)-F(x) \leq_{K}(y-x)
$$

if $x \leq_{K} y$. By (4.4),

$$
g^{\prime \prime}(t)=\frac{1}{(1-t)^{3}}, t \in(-\infty, 0),
$$

which implies that $g^{\prime \prime}(t)>0$ for all $t \in(-\infty, 0)$. Hence $g(t)$ is convex on $(-\infty, 0)$ and furthermore

$$
\left.g\left(\lambda t_{1}+(1-\lambda) t_{2}\right) \leq \lambda g\left(t_{1}\right)+(1-\lambda) g\left(t_{2}\right)\right)
$$

for all $t_{1}, t_{2} \in(-\infty, 0)$ and $\lambda \in[0,1]$. Similarly,

$$
\left.h\left(\lambda t_{1}+(1-\lambda) t_{2}\right) \leq \lambda h\left(t_{1}\right)+(1-\lambda) h\left(t_{2}\right)\right)
$$

for all $t_{1}, t_{2} \in(-\infty, 0)$ and $\lambda \in[0,1]$. Summarizing (4.6) and (4.7), we get

$$
\begin{aligned}
F(\lambda x+(1-\lambda) y) & =\left(g\left(\lambda x_{1}+(1-\lambda) y_{1}\right), h\left(\lambda x_{2}+(1-\lambda) y_{2}\right)\right) \\
& \leq_{K}\left(\lambda g\left(x_{1}\right)+(1-\lambda) g\left(y_{1}\right), \lambda h\left(x_{2}\right)+(1-\lambda) h\left(y_{2}\right)\right) \\
& =\lambda F(x)+(1-\lambda) F(y)
\end{aligned}
$$

for all $\lambda \in[0,1]$ and every pair of distinct comparable points $x, y \in \Omega$, implying that $F(x)$ is convex in $K$-order on $\Omega$. Thus the claim is proved. Since

$$
-\frac{1}{9} M^{2}+\frac{10}{9} M-1=-\frac{1}{9}(M-5)^{2}+\frac{16}{9} \geq 0
$$

for all $M \in[1,9]$, i.e., inequality (4.1) holds for any $M \in[1,9]$, by Theorem 4.1 we see that Eq.(4.3) has a convex solution $f \in C_{c v}^{+}(\Omega, 0, M)$.

We end the paper with some remarks. The same difficulties as in [5] are encountered, hence, we did not discuss decreasing convex (resp. concave) solutions for Eq.(1.1) and the existence of decreasing (resp. increasing) solutions for given $F \in C^{+}\left(\Omega, 0, M_{1}\right)$ (resp. $\left.C^{-}\left(\Omega, 0, M_{1}\right)\right)$ and the existence of increasing convex (resp. concave) solution for given $F \in C_{c c}^{+}\left(\Omega, 0, M_{1}\right)$ (resp. $\left.C_{c v}^{+}\left(\Omega, 0, M_{1}\right)\right)$ are not given yet for Eq.(1.1).

Acknowledgment. The author is most grateful to the anonymous referee for the careful reading of the manuscript and valuable suggestions. 


\section{Appendix: Schauder-Tychonoff Theorem}

Schauder-Tychonoff Theorem ([1, Theorem 8.2, p. 96]). Let E be a Hausdorff locally convex linear topological space, $C$ a convex subset of $E$ and $F$ : $C \rightarrow E$ a continuous mapping such that $F(C) \subseteq A \subseteq C$ with $A$ compact. Then $F$ has at least one fixed point.

\section{References}

[1] R. P. Agarwal, M. Meehan, and D. O'Regan, Fixed Point Theory and Applications, Cambridge University Press, Cambridge, 2001.

[2] H. Amann, Fixed point equations and nonlinear eigenvalue problems in ordered Banach spaces, SIAM Rev. 18 (1976), no. 4, 620-709.

[3] K. Baron and W. Jarczyk, Recent results on functional equations in a single variable, perspectives and open problems, Aequationes Math. 61 (2001), no. 1-2, 1-48.

[4] J. G. Dhombres, Itération linéaire d'ordre deux, Publ. Math. Debrecen 24 (1977), no. $3-4,277-287$.

[5] X. Gong and W. Zhang, Convex solutions of the polynomial-like iterative equation in Banach spaces, Publ. Math. Debrecen 82 (2013), no. 2, 341-358.

[6] W. Jarczyk, On an equation of linear iteration, Aequationes Math. 51 (1996), no. 3, 303-310.

[7] M. Kuczma, B. Choczewski, and R. Ger, Iterative Functional Equations, Encyclopedia Math. Appl., vol. 32, Cambridge Univ. Press, Cambridge, 1990.

[8] M. Kuczma and A. Smajdor, Fractional iteration in the class of convex functions, Bull. Acad. Pol. Sci. Sér. Sci. Math. Astron. Phys. 16 (1968), 717-720.

[9] M. Kulczycki and J. Tabor, Iterative functional equations in the class of Lipschitz functions, Aequationes Math. 64 (2002), no. 1-2, 24-33.

[10] J. Mai and X. Liu, Existence, uniqueness and stability of $C^{m}$ solutions of iterative functional equations, Sci. China Ser. A 43 (2000), no. 9, 897-913.

[11] M. Malenica, On the solutions of the functional equation $\phi(x)+\phi^{2}(x)=F(x)$, Mat. Vesnik 6(19)(34) (1982), no. 3, 301-305.

[12] J. Matkowski and W. Zhang, On linear dependence of iterates, J. Appl. Anal. 6 (2000), no. 1, 149-157.

[13] A. Mukherjea and J. S. Ratti, On a functional equation involving iterates of a bijection on the unit interval, Nonlinear Anal. 7 (1983), no. 8, 899-908.

[14] - A functional equation involving iterates of a bijection on the unit interval. II, Nonlinear Anal. 31 (1998), no. 3-4, 459-464.

[15] W. Rudin, Functional Analysis, Second edition, McGraw Hill, New York, 1991.

[16] J. Si, Existence of locally analytic solutions of the iterated equation $\sum_{i=1}^{n} \lambda_{i} f^{i}(x)=$ $F(x)$, Acta Math. Sinica. 37 (1994), no. 5, 590-600.

[17] J. Tabor and J. Tabor, On a linear iterative equation, Results Math. 27 (1995), no. 3-4, $412-421$.

[18] J. Tabor and M. Z̈ołdak, Iterative equations in Banach spaces, J. Math. Anal. Appl. 299 (2004), no. 2, 651-662.

[19] G. Targonski, Topics in Iteration Theory, Studia Mathematica: Skript, 6. Vandenhoeck \& Ruprecht, G̈̈tingen, 1981.

[20] T. Trif, Convex solutions to polynomial-like iterative equations on open intervals, Aequationes Math. 79 (2010), no. 3, 315-325.

[21] B. Xu and W. Zhang, Decreasing solutions and convex solutions of the polynomial-like iterative equation, J. Math. Anal. Appl. 329 (2007), no. 1, 483-497.

[22] D. Yang and W. Zhang, Characteristic solutions of polynomial-like iterative equations, Aequationes Math. 67 (2004), no. 1-2, 80-105. 
[23] E. Zeidler and P. R. Wadsack, Nonlinear Functional Analysis and Its Applications, Springer-Verlag, New york, 1986.

[24] J. Zhang, L. Yang, and W. Zhang, Some advances on functional equations, Adv. Math. (China) 24 (1995), no. 5, 385-405.

[25] W. Zhang, Discussion on the iterated equation $\sum_{i=1}^{n} \lambda_{i} f^{i}(x)=F(x)$, Chinese Sci. Bull. 32 (1987), no. 21, 1444-1451.

[26] Discussion on the differentiable solutions of the iterated equation $\sum_{i=1}^{n} \lambda_{i} f^{i}(x)$ $=F(x)$, Nonlinear Anal. 15 (1990), no. 4, 387-398.

[27] Solutions of equivariance for a polynomial-like iterative equation, Proc. Roy. Soc. Edinburgh Sect. A 130 (2000), no. 5, 1153-1163.

[28] W. Zhang, K. Nikodem, and B. Xu, Convex solutions of polynomial-like iterative equations, J. Math. Anal. Appl. 315 (2006), no. 1, 29-40.

[29] L. Zhao, A theorem concerning the existence and uniqueness of solutions of the functional equation $\lambda_{1} f(x)+\lambda_{2} f^{2}(x)=F(x)$, J. Univ. Sci. Tech. 32 (1983), 21-27 (in Chinese).

Key Laboratory of Numerical Simulation of Sichuan Province Neijiang, Sichuan 641100, P. R. China

AND

College of Mathematics and Information Science

NeiJiang Normal University

Neijiang, Sichuan 641100, P. R. China

E-mail address: xbgong@163.com 